\title{
General and facile purification of dye-labeled oligonucleotides by $\mathrm{pH}$-controlled extraction
}

So Young Bak ${ }^{1, *}$, Jihee Hwang ${ }^{1, *}$, Sohyeon Bae ${ }^{1}$, Soonkyu Lim², Younggyu Kim², Khalid Alzahrani ${ }^{3}$, Rizwan Wahab ${ }^{3}$, Abdulaziz Alkhedhairy $^{3}$, and Seong Keun Kim ${ }^{1}$

'Department of Chemistry, Seoul National University, Seoul, Republic of Korea, ${ }^{2}$ Department of Research and Development, LumiMac, Inc., Seoul, Republic of Korea, and College of Sciences, King Saud University, Riyadh, Saudi Arabia

*S.Y.B. and J.H. contributed equally to this work.

BioTechniques 64:21-23 (January 2018) doi 10.2144/000114627

Keywords: dye-labeled oligonucleotide purification; free dyes extraction; fluorescent dye $\mathrm{pK}_{\mathrm{a}}$

Supplementary material for this article is available at www.BioTechniques.com/article/114627.

Previously, we reported a method for facile purification of oligonucleotides labeled with hydrophobic dyes, based on the solubility difference between the hydrophilic DNA and unreacted dye. Here, we present a new purification method applicable to any dye regardless of its hydrophobicity. We exploited the population shift of a fluorescent dye in a low-pH aqueous solution from its anionic form toward its neutral form. When the $\mathrm{pH}$ of an aqueous solution containing dye-labeled DNA and unreacted free dye is lowered, and the solution is mixed with a hydrophobic organic solvent (butanol), the neutral free dye is preferentially dissolved in the organic phase, leaving behind the hydrophilic dye-labeled DNA in the aqueous phase. We experimentally verified that our new method results in high yields of dye-labeled oligonucleotides and the efficient removal of free dye.

Alongside recent advances in fluorescence microscopy technology, a growing number of fluorescent molecules have been developed to label various targets. The most important step in the dye-labeling procedure is removal of the unreacted fluorescent dye to enhance the signalto-noise ratio in the detection of the targeted molecule. Although removal of unreacted dye is of paramount importance, only a few methods exist for purification of dye-labeled oligonucleotides. Among these, HPLC, gel electrophoresis, size exclusion chromatography, and ethanol precipitation are widely used, but they are often timeconsuming and inefficient, leading to a considerable loss of labeled sample during the extraction process (1).
The difference in the solubility of the free dye itself and the dye-labeled oligonucleotides has also been utilized to enable facile separation using mixtures of two immiscible liquid phases (2).Recently, we employed this purification approach using butanol to extract hydrophobic dyes such as ATTO from dye-labeled DNA, which would remain in the aqueous phase (3). Although a much-improved technique compared with ethanol precipitation, it is applicable only to hydrophobic fluorescent dyes in order to take maximum advantage of the solubility difference between the dye and hydrophilic DNA. Here, we present a new method that is still facile and efficient but is also applicable to any fluorescent dye-regardless of its hydrophobicity-because it exploits the $\mathrm{pH}$-dependent solubility of the dye in aqueous solution.

Figure 1A shows the drastic solubility differences of a free dye in aqueous solutions of different $\mathrm{pH}$ values. The free dye (Cy5) and dye-labeled DNA remain mostly together in the aqueous phase (bottom layer of the left vial) at pH 8.5 but become separated between the butanol and aqueous phases at $\mathrm{pH} 3.0$ (top and bottom layer of the right vial, respectively). It should be noted that $\mathrm{pH} 8.5$ is a typical labeling buffer condition for a primary aminebased DNA labeling reaction, with the optimum $\mathrm{pH}$ range for the $\mathrm{N}$-hydroxysuccinimide (NHS)-ester reaction being $\mathrm{pH}$ 8.0-9.0. The labeling buffer we typically used was $0.1 \mathrm{M}$ sodium borate.

$\mathrm{pH}$-controlled butanol extraction was examined for various classes of fluorescent dyes: cyanine [Cy3 (Cat. \#PA 13101; GE Healthcare, Chicago, IL), Cy3B (Cat. \#PA 63100), Cy5 (Cat. \#PA 15104)], oxazine (Nile Red) (Cat. \#N0659, TCl, Tokyo, Japan), and rhodamine [rhodamine 6G (Cat. \#252433; Sigma Aldrich, St. Louis, MO), ATTO 633 (Cat. \#AD 633-31; ATTO-TEC GmbH, Siegen, Germany), Alexa 488 (Cat. \#A20000; Thermo

\section{METHOD SUMMARY}

Our new method for the purification of dye-labeled DNA is based on an increase in the neutral form of the fluorescent dye at lower $\mathrm{pH}$, allowing the dye to better dissolve in the hydrophobic organic layer of an immiscible two-phase solution. The hydrophilic DNA is then recovered from the aqueous phase. 
Fisher Scientific, Waltham, MA)]. We were able to separate the free dyes into the butanol phase by controlling the $\mathrm{pH}$ of the aqueous solution (Supplementary Figures S1 and S2).

The relationship between the $\mathrm{pH}$ of an aqueous solution and the solubility of a molecule in that solution has been noted in an earlier study (4). The $\mathrm{pH}$-dependent solubility can be understood using the Henderson-Hasselbalch equation:

$$
p H=p K_{a}+\log _{10}\left(\frac{\left[A^{-}\right]}{[H A]}\right) .
$$

According to the equation, the negatively charged form of the molecule is the dominant species in aqueous solution when the $\mathrm{pH}$ of the solution is greater than the $\mathrm{pK}_{\mathrm{a}}$ of the dye, while the molecule will increasingly react with a proton at lower $\mathrm{pH}$ values to form the neutral species that is more soluble in the hydrophobic organic solvent.

The net result is that the negatively charged dye molecule is effectively transferred from the aqueous phase to the butanol phase as it is converted from the anionic to neutral form at lower $\mathrm{pH}$. Of course, DNA remains in the aqueous phase due to the negative charges on its phosphate groups, with a $\mathrm{pK}_{\mathrm{a}}$ of 0.7-1.0 (5), as long as the solution's $\mathrm{pH}$ is not reduced below the point where the phosphate groups themselves become neutralized.

In order to confirm the $\mathrm{pK}_{\mathrm{a}}$ values of the dyes and the DNA, the absorbance of free dye was measured in butanol after purification using a UV/ Vis spectrometer, while the $\mathrm{pH}$ of the aqueous solution was varied from 1.0 to 9.0 (Figure 1B and Supplementary Table S2). The Boltzmann function was fitted to each curve, and the $\mathrm{pH}$ at the half-point of absorbance (where half of the free dye molecules exist as $\left[\mathrm{A}^{-}\right]$ and the other half as $[\mathrm{HA}]$ ) is defined to be the $\mathrm{pK}_{\mathrm{a}}$. The measured $\mathrm{pK}_{\mathrm{a}}$ is consistent with the net charge of each dye, as can be seen in the case of Alexa 488 , which has a net charge of -3.94 at $\mathrm{pH} 7.4$ and showed the lowest $\mathrm{pK}_{\mathrm{a}}$ of 3.1 (6). The $\mathrm{pK}_{\mathrm{a}}$ values of free Cy 5 and DNA were measured as 4.8 and 1.6, respectively, so we can achieve purification for Cy5-labeled DNA with an aqueous solution of $\mathrm{pH}$ 3.0. Purifi-
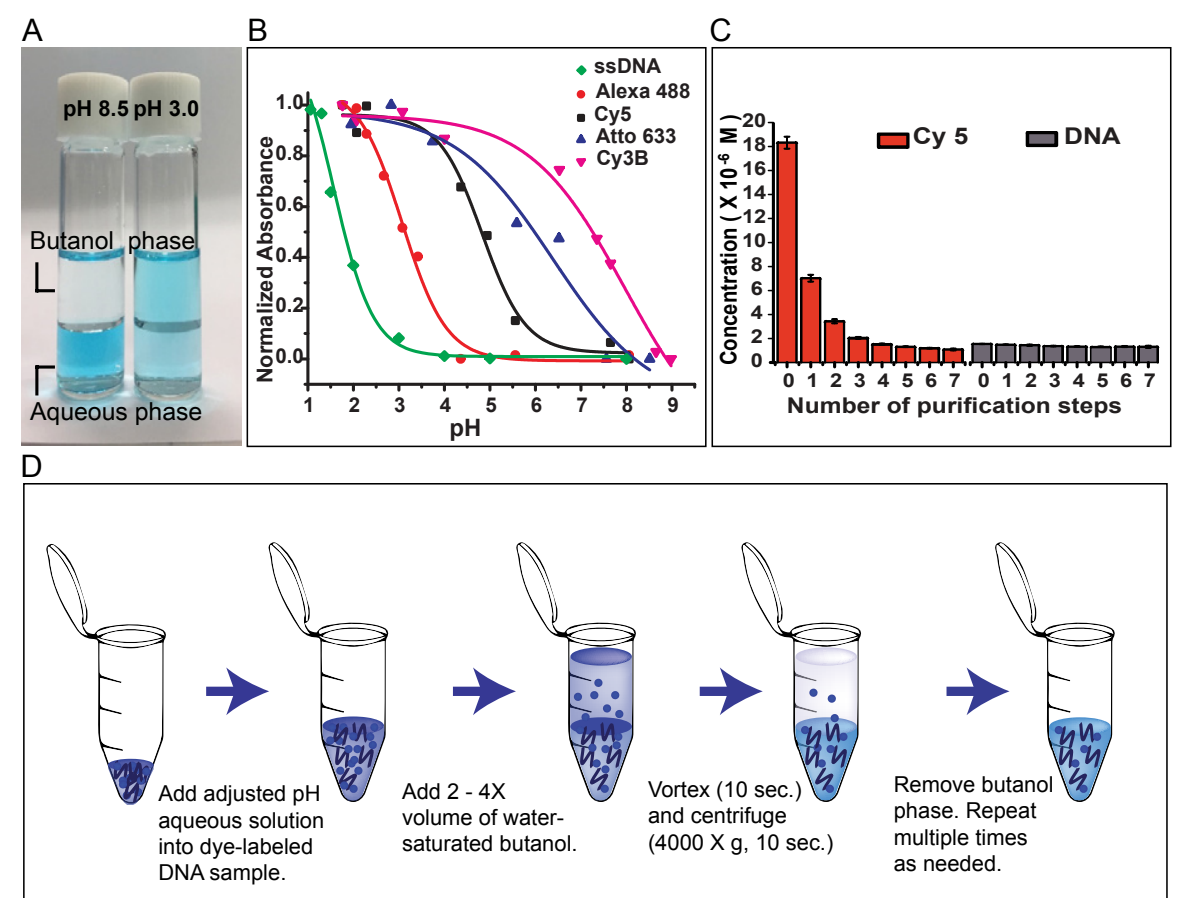

Figure 1. $\mathrm{pH}$-controlled extraction of free $\mathrm{Cy} 5$ dye from Cy5 dye-labeled oligonucleotides in aqueous buffer. (A) Mixture of free Cy5 dye and Cy5 dye-labeled DNA oligonucleotides (60 bp) in an immiscible binary phase solution of butanol and water at pH 8.5 (left) and pH 3.0 (right). (B) Normalized absorbance of aqueous solutions of the dyes Alexa 488, Cy5, ATTO 633, and Cy3B, as well as oligonucleotides (60 bp), as a function of $\mathrm{pH}$ from $\mathrm{pH} 1.0$ to 9.0. (C) Purification of Cy5-labeled DNA at $\mathrm{pH} 3.0$ showing efficient removal of the free $\mathrm{Cy} 5$ dye. (D) $\mathrm{pH}$-controlled extraction procedure. The appropriate $\mathrm{pH}$ for the labeling buffer, depending on the $\mathrm{pK}_{\mathrm{a}}$ of each fluorescent dye, is obtained by adding $0.5 \mathrm{M}$ or $1 \mathrm{M} \mathrm{HCl}$. The $\mathrm{pH}$-adjusted buffer is added to the DNA sample, followed by the addition of water-saturated butanol. After vortexing vigorously for $10 \mathrm{~s}$, the mixture is separated into aqueous and butanol phases by a brief centrifugation $(4000 \times g, 10 \mathrm{~s})$. Free dye is removed by discarding the butanol phase. The purification steps can be repeated as many times as needed.
A

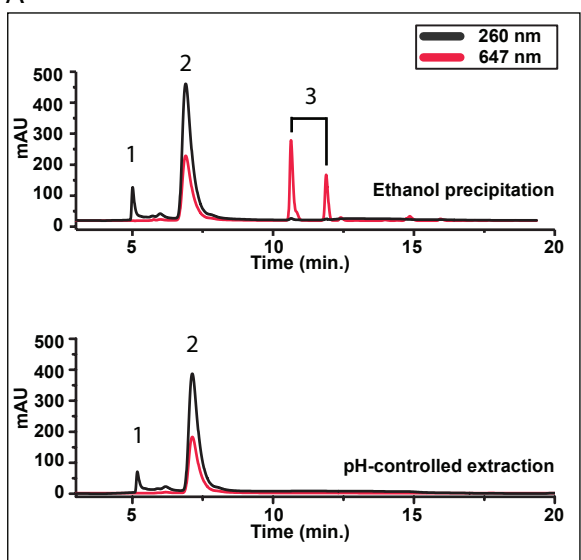

B

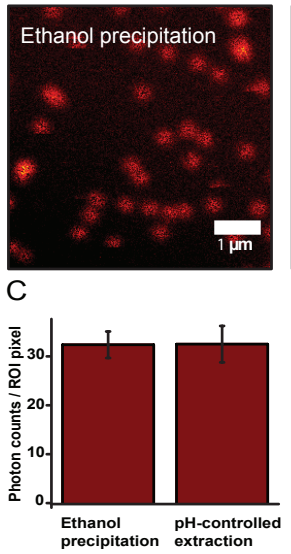

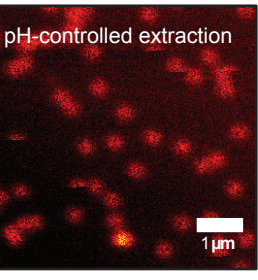

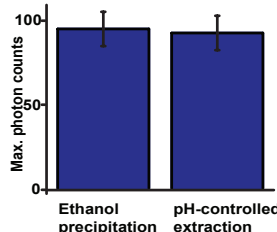

Figure 2. Comparison of DNA samples prepared by ethanol precipitation versus $\mathrm{pH}$-controlled extraction. (A) Cy5-labeled oligonucleotides were analyzed by HPLC with a C184.6/100 reverse phase column (ZORBAX Eclipse Plus 95Å C18 Eclipse, Cat. \#PN 959961-902; Agilent Technologies, Santa Clara, CA). The running conditions were 100\% Buffer D up to 2 min, followed by gradually increasing Buffer $A$ to $80 \%$ for 40 min. Buffers $D$ and $A$ were $50 \mathrm{mM}$ triethylammonium acetate (TEAA)/acetonitrile $(A C N)$ and $100 \% A C N$, respectively. Flow rate was $1 \mathrm{~mL} / \mathrm{min}$. The results show the presence of free dye (Peak 3; $647 \mathrm{~nm}$ spectrum between 10 and $13 \mathrm{~min}$ ) by ethanol precipitation (top) versus its complete removal by $\mathrm{pH}$-controlled extraction at $\mathrm{pH} 3.0$ (bottom). Peak 1: unlabeled DNA; Peak 2: dye-labeled DNA. (B) Confocal images of doublestranded DNA (dsDNA) labeled with Cy5 and immobilized on a glass surface via biotin-NeutrAvidin interaction. (C) Ratio of photon counts versus region of interest (ROI) pixels (left panel) and maximum photon counts (right panel) of the confocal images shown in (B). ROI was selected for each fluorescent molecule, and the photon counts and maximum photon counts were measured. 
cation efficiency at $\mathrm{pH} 3.0$ is much greater than that at $\mathrm{pH}$ 8.5. (Figure $1 \mathrm{C}$ and Supplementary Figure S3).

The purification process is simple and fast (Figure 1D). The entire procedure takes $<10 \mathrm{~min}$, which is faster than any other dye-labeled oligonucleotide purification method. After purification, the sample can be titrated back to neutral $\mathrm{pH}$ for storage or further treatment by adding an alkaline solution such as $\mathrm{NaOH}$.

Single-stranded DNA (ssDNA) labeled with Cy5 was purified using both ethanol precipitation and our new method, and then separated by HPLC. The HPLC data clearly demonstrate superior removal of unincorporated dye when using our method compared to the ethanol precipitation technique (Figure 2A). The data also prove that the amide bond between the DNA and fluorescent dye is intact.

To test the robustness of samples prepared by our new method for use in downstream assays, purified dye-labeled ssDNA was annealed with a complementary strand for confocal imaging. An analysis was carried out for each individual molecule of Cy5-labeled double-stranded DNA (dsDNA) (Figure 2B), which showed that there was no difference in the photon counts per pixel and the maximum photon counts between samples prepared by the ethanol precipitation method and the $\mathrm{pH}$-controlled extraction method, verifying that these fluorescence properties are not altered by the $\mathrm{pH}$ shift (Figure 2C). We also confirmed that DNA samples prepared by our new $\mathrm{pH}$-controlled method do not show any noticeable signs of degradation, such as fragmentation or depurination, based on native PAGE gel and HPLC data (Supplementary Figures S4 and S5). Furthermore, our experimental data showed a $97 \%$ recovery yield for the new method, which is higher than the $70 \%-95 \%$ recovery yield of gel filtration (IIlustra NAP-10 Columns, Cat. \#17-0854-02; GE Healthcare, Chicago, IL).

\section{Author contributions}

S.Y.B. performed the main experiments, which were designed by S.Y.B., J.H., and Y.K. S.B. performed the confocal microscopy experiments and data analysis, while S.L. performed various solvent experiments. K.A., R.W., and A.A. shared their expertise on separation techniques. S.Y.B. wrote the manuscript. S.K.K. supervised the study and corrected the manuscript.

\section{Acknowledgments}

We would like to thank the TRE program of King Saud University for support.

\section{Competing interests}

The authors declare no competing interests.

\section{References}

1. Sambrook, J. and D.W. Russell. 2001. Molecular Cloning: A Laboratory Manual. Cold Spring Harbor Laboratory Press, Cold Spring Harbor, NY.

2. Kirby, K.S. 1957. A new method for the isolation of Deoxyribonucleic Acids: Evidence on the nature of bonds between Deoxyribonucleic acid and protein. Biochem. J. 66:495-504.
3. Hwang, J., J. Kang, S. Kim, and Y. Kim. 2013. A convenient and efficient purification method for chemically labeled oligonucleotides. Biotechniques 54:280-282.

4. Kolmakov, K., C.A. Wurm, D.N. Meineke, F. Göttfert, V.P. Boyarskiy, V.N. Belov, and S.W. Hell. 2014. Polar red-emitting rhodamine dyes with reactive groups: synthesis, photophysical properties, and two-color STED nanoscopy applications. Chem Eur J. 20:146157.

5. Shabarova, Z.A. and A.A. Bogdanov. 2008. Advanced Organic Chemistry of Nucleic Acids: Ionization of The Phosphate Group. John Wiley \& Sons, Hoboken, NJ.

6. Zanetti-Domingues, L.C., C.J. Tynan, D.J. Rolfe, D.T. Clarke, and M. MartinFernandez. 2013. Hydrophobic fluorescent probes introduce artifacts into single molecule tracking experiments due to non-specific binding. PLoS One 8:e74200.

Received 20 June 2017; accepted 15 November 2017.

Address correspondence to Seong Keun Kim, Department of Chemistry, Seoul National University, Seoul, Republic of Korea. E-mail: seongkim@snu.ac.kr

To purchase reprints of this article, contact: biotechniques@fosterprinting.com

\section{New Era in Single Cell Genomics}

Single Cell RNA Analysis Using Drop-Seq Technology

- Highly Parallel Cell \& Mol Bar Code DNA Coated Beads (fig.1);Lead to Single Cell Transcriptomes (STAMPS)

- Capable of High Level RNA Capture, rt PCR \& cDNA Library (fig.2); Macosko et al., Cell 2015, 161 (5), 1202-1214, Gierahn et. al., Nature Methods 13 Feb 2017.

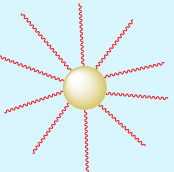

Fig. 1

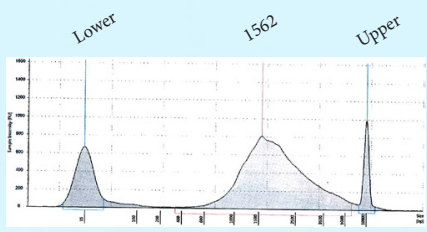

Fig. 2

\section{- A multiple particle type of Bar Code Beads are} now available.

Your source of Barcode Beads confidently from ChemGenes

UNPARALLED Expertise in

NUCLEIC ACID Chemistry since 1981

ISO 9001:2008 Certified Company

\section{ChemGenes Toll free: (800) 762-9323}

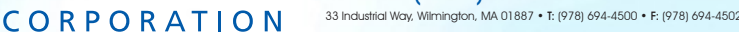

For more information, visit us at www. ChemGenes.com 